\title{
Menopause uncertainty: the impact of two educational interventions among women during menopausal transition and beyond
}

\author{
Fateme Afshari ${ }^{1}$, Narjes Bahri², Moosa Sajjadi ${ }^{3}$, Mohammad Reza Mansoorian4, Hamid Reza Tohidinik ${ }^{5,6}$ \\ ${ }^{1}$ Department of Medical Surgical Nursing, School of Nursing, Gonabad University of Medical Science, Gonabad, Iran \\ 2Department of Midwifery, Faculty of Medicine, Social Development and Health Promotion Centre, Gonabad University of Medical \\ Sciences, Gonabad, Iran \\ ${ }^{3}$ Department of Medical Surgical Nursing, School of Nursing, Social Development and Health Promotion Centre, Gonabad University \\ of Medical Sciences, Gonabad, Iran \\ ${ }^{4}$ Department of Management and Community Health Nursing, Gonabad University of Medical Sciences, Gonabad, Iran \\ ${ }^{5} \mathrm{HIV} / \mathrm{STI}$ Surveillance Research Center, and WHO Collaborating Center for HIV Surveillance, Institute for Futures Studies in Health, \\ Kerman University of Medical Sciences, Kerman, Iran \\ ${ }^{6}$ Department of Epidemiology and Biostatistics, School of Public Health, Tehran University of Medical Sciences, Tehran, Iran
}

\begin{abstract}
Introduction: Menopause is a natural part of women's lives, which is associated with physical and mental changes and can lead to uncertainty and consequent undesirable effects in the lives of menopausal women. The present study was conducted to compare the impact of multimedia and booklet educational methods on the uncertainty about menopause during this transition period.

Materials and methods: In a cluster randomized trial 68 women aged 45-55 years were randomly allocated into two groups: multimedia and booklet. Data were collected using knowledge and uncertainty questionnaires about menopause in three stages, i.e. before intervention, immediately after intervention (two weeks later), and one month later. Repeated-measures analysis of variance (rANOVA) was used to compare knowledge and uncertainty scores between two groups for the duration of the study. Data were analysed using SPSS software and $p$ values $<0.05$ were considered as statistically significant.

Results: The results of the study indicated a high level of uncertainty in both groups before the implementation of the educational program. There was no significant difference between groups in terms of knowledge and uncertainty about menopause over time $(p>0.05)$. However, we found a significant increase in knowledge and a significant decrease in uncertainty scores in both groups $(p<0.001)$ after the interventions.

Conclusions: Although the findings of this study did not show a significant difference between the effect of multimedia and booklet educational methods in postmenopausal women, the high level of uncertainty of women before intervention and its significant reduction after the implementation of the educational programs indicated the importance of education throughout this period.
\end{abstract}

Key words: illness uncertainty, distance education, multimedia, booklet, menopause, menopausal transition.

\section{Introduction}

The number of postmenopausal women worldwide will reach 1.2 billion by 2030, and it is expected that by 2020 about 5 million postmenopausal women will live in Iran [1]. Women experience specific physical and mental changes during the transition period to menopause [2-4]. Although much of the information women receive about menopause helps them better understand the vague and complex symptoms of menopause and have a good assessment of their "normal" experiences, often, because of receiving conflicting information, they are confused and face uncertainty regarding different recommendations about the management and treatment of symptoms and the prevention of complications [5, 6].

The concept of "uncertainty" was first introduced by Mishel in 1981. She states that "uncertainty in illness" occurs when the patient is not able to determine the meaning of illness-related events. Therefore, it is considered an important psychological stressor for the patient, which can leave major effects on mental compliance and illness outcomes [7, 8].

Because menopause is a physiological phenomenon and is not considered pathological, it seems that the 
concept of uncertainty in illness cannot be fully applied to it. But in some ways, it seems that this theory can also be generalised to menopause because during the transition to menopause, women encounter high degrees of ambiguity and variability of symptoms [4]. Studies have shown that women feel concerned after their first confrontation with menopausal signs and symptoms, and they have doubts about their menopausal condition, the nature and severity of their symptoms, and their ability to overcome them $[9,10]$. These worries and concerns are a symbol of the concept of Mishel uncertainty theory.

The results of some qualitative studies have also shown that the concept of uncertainty exists in the area of menopause and that the experiences associated with menopause in women are so variable and unpredictable that they can move the person to uncertainty [11-13]. On the other hand, conflicting ideas and contradictory information provided by healthcare providers and other people, including friends and relatives, may create ambiguity along with uncertainty $[1,14]$. In some cases, even the awareness of certain menopausal symptoms, such as the risk of osteoporosis, may cause uncertainty and concern in some women [15]. Another important area of uncertainty about menopause is the issue of hormone therapy, which is a challenging topic. The existence of conflicting evidence about the benefits and dangers of hormone therapy can cause uncertainty in women when making decisions for hormone therapy $[16,17]$.

However, uncertainty about menopause can be associated with negative outcomes, including an increase in the severity of menopause symptoms or negative attitude toward menopause [18, 19]. Previous studies on uncertainty in illnesses have shown that the degree of uncertainty in an illness is clearly related to the level of knowledge of individuals about the illness. For example, Pie et al. showed that there is a significantly negative relationship between uncertainty and level of knowledge about menopause, and that educational interventions to increase the level of women's awareness can reduce uncertainty in postmenopausal women [18].

There are also other studies that emphasise the link between inadequate knowledge and high levels of uncertainty in illnesses such as cancer, endometriosis, heart disease, and liver disease [20-23]. Therefore, one of the strategies to deal with uncertainty is the implementation of educational interventions to raise awareness [7, 21, 23]. Today, distance education methods, such as multimedia and booklets, have been more welcomed by learners [7, 24, 25]. Findings of previous studies show the positive effects of these two methods on increasing self-care activities, awareness, increasing self-efficacy, and reducing anxiety in patients [24-27].
Because the health system in Iran does not provide specific care associated with menopause, and women in the menopausal transition do not have regular visits to health centres, the feasibility of using participative methods in this population is low; it seems that the use of distance education is more appropriate for them. Therefore, this study was conducted aimed at investigating the comparison of the effectiveness of multimedia and booklets on uncertainty about menopause among postmenopausal women.

\section{Material and methods \\ Trial design}

The present study was a cluster randomized trial with two parallel arms and an allocation ratio of $1: 1$. The study protocol was approved by the Research Council of Gonabad University of Medical Sciences, Gonabad, Iran (grant number: 93/4/P). The study was also registered in IRCT (registration ID: IRCT20171025037003N2). This trial was reported based on the CONSORT statement 2010 checklist [28].

\section{Study setting}

The study was conducted from May 2017 to August 2018 in Gonabad, a city located in the northeast of Iran. Gonabad is a small and relatively traditional city in Khorasan-Razavi province and the $13^{\text {th }}$ in population ranking of this province. At the time of the study, the number of female residents aged between 45-60 years was around 8221 [29]. Moreover, there was no special service to be provided for menopausal women in the national health system of Iran at the time.

\section{Participants and sample size}

The participants were 45 to 60-year-old women with at least elementary education. These women did not have early menopause and had no history of hysterectomy or ovariectomy. They did not have any chronic psychiatric illness or neurological disorders or hearing impairment, and also had not completed a threemonth pre-menopausal specialist training program prior to the study. Moreover, the participants in the multimedia group had access to a computer at home and had the skills to use multimedia. The exclusion criteria included the reluctance to continue to collaborate in the study, the lack of full implementation of the multimedia educational program or not using the booklet, and the receipt of any other educational program related to menopause during the stages of the study.

The sample size was calculated as 27 by using the mean and standard deviation of the uncertainty score 
in Lasker et al. [21]. The test power and confidence interval $(\mathrm{Cl})$ were considered as $90 \%$ and $95 \%$, respectively. Ultimately, by taking into account the probability of the loss of a number of participants in the study, 40 women were included in each group -80 in total.

\section{Sampling and randomisation}

Multi-stage random sampling was done. At first, two centres (a centre for the multimedia group and a centre for the booklet group) from among all the community health centres of Gonabad were selected randomly for intervention. Subsequently, 40 individuals were randomly selected in each centre using a sampling framework prepared based on household census data.

\section{Study instruments}

In order to collect data, a demographic questionnaire, a menopausal knowledge questionnaire, and an uncertainty questionnaire for menopause were used. The demographic questionnaire included variables such as age, occupation, educational level, number of children, number of pregnancies, age of menarche, and last menstrual period.

The menopausal knowledge questionnaire was designed by the researchers. It contained 26 sentences about menopause, with three options: true, false, and I do not know. The more points obtained, the higher the awareness of menopause. To determine the content validity of the questionnaire, 10 faculty members reviewed it. Some revisions were done based on their opinions, and finally it was approved. The reliability of this scale was assessed using Cronbach's $\alpha$ method, which was 0.9.

The uncertainty questionnaire for menopause is a modified version of Mishel's Uncertainty in Illness Questionnaire. In this study, the modified version of Mishel's Uncertainty Questionnaire (community form MUIS-C) was used. This version of the questionnaire was designed for non-hospitalised individuals and includes 23 questions on a five-point Likert scale (totally agree, agree, neither agree nor disagree, disagree, totally disagree). Each statement has one to five points, and the total score of the questionnaire is between 23 and 115 points. Lemaire and Lens used this version in 1995, after making some changes, to assess uncertainty in menopause [14]. In their study, the reliability of the adjusted uncertainty questionnaire in menopause using Cronbach's a was $r=0.9$. In this study, the validity and reliability of this questionnaire were evaluated. The questionnaire was then reviewed by 10 faculty members after translation into Persian to determine the validity of its content, and after approval of the revisions, the questionnaire was finalised. The reliability of this questionnaire was also assessed using Cronbach's $\alpha$ method, which was 0.79 .

\section{Intervention and outcomes}

After calling the selected individuals at the sampling stage and ensuring that they met the inclusion criteria, the researchers invited them to participate in the study. At the beginning of the study in each centre and for each intervention group, a separate meeting was held, in which a brief description of the research objectives and how it was to be done was presented. Next the participants received written and informed consent forms, and completed the demographic questionnaire, the menopausal knowledge questionnaire, and the uncertainty about menopause questionnaire.

In the multimedia group, CDs with educational content related to menopause including physiology of menopause, signs and symptoms of menopause, various ways to treat menopausal symptoms and self-care education during menopause were distributed to the participants. The compilation of the CD's scientific content was done using the latest reference books, as well as the contents of the booklets and educational material published by the Ministry of Health and Medical Education of Iran, and it was approved by a number of reproductive health professionals. The CDs included a combination of movies, animations, images, and sounds that were provided by a computer engineer and supervised by the researchers. After giving one CD to each participant, information was given on how to use it, and a two-week timetable was presented to the participants for viewing it. During these two weeks, the researchers reminded the participants by texting them. The women were also provided with a telephone number to contact the researchers in case they had questions or encountered any problems.

In the booklet group, the participants were given a booklet with content exactly the same as the multimedia package and were asked to read the content within two weeks. Likewise, a telephone number was given to them in the event of a problem or question. Immediately after the end of the two weeks and also one month later, the menopausal knowledge questionnaire and the uncertainty questionnaire for menopause were once again completed by all the participants.

\section{Statistical analysis}

Continuous data were reported as mean \pm SD. The normality of data distributions was tested using the one-sample Kolmogorov-Smirnov test. Independent samples $t$-test and $\chi^{2}$ test were performed to compare continuous and categorical baseline variables, respectively. We applied repeated-measures analysis of vari- 
ance (rANOVA) to compare knowledge and uncertainty scores in two groups for the duration of the study and to check time-group interaction. Follow-up analyses included an independent samples t-test at each time point. An intention to treat (ITT) approach was followed in the analysis, and the level of significance was set at $p<0.05$. All analyses were carried out using SPSS 16 (SPSS Inc., Chicago, IL).

\section{Ethical considerations}

The protocol of the study was approved by the Regional Committee on Medical Ethics of Gonabad University of Medical Sciences (registration code: IR.GMU. REC.1396.61). All participants provided written, informed consent to participate in the study.

\section{Results}

Although 80 women were initially recruited, 13 women were excluded (five from the multimedia group and seven from the booklet group), and 68 women (35 in the multimedia group and 33 the in-booklet group) completed the study (Fig. 1).

The two groups had no significant differences in demographic characteristics such as age, education, job, age at menarche, number of children, and last menstrual period $(p>0.05)$ (Table 1$)$.

There was no significant difference between the two groups in terms of knowledge about menopause before the intervention $(p=0.32)$. The rANOVA showed no difference between the two groups in terms of knowledge score $(p=0.34)$, but there was a significant increase in the score of knowledge about menopause over time $(p<0.001)$. There was no evidence for timegroup interaction ( $p=0.93$ ) (Fig. 2). Comparing each time point together, there was no significant difference between the two groups in terms of knowledge about menopause immediately after and one month after intervention (Table 2).

Uncertainty about menopause was measured before, immediately after, and one month after interven-

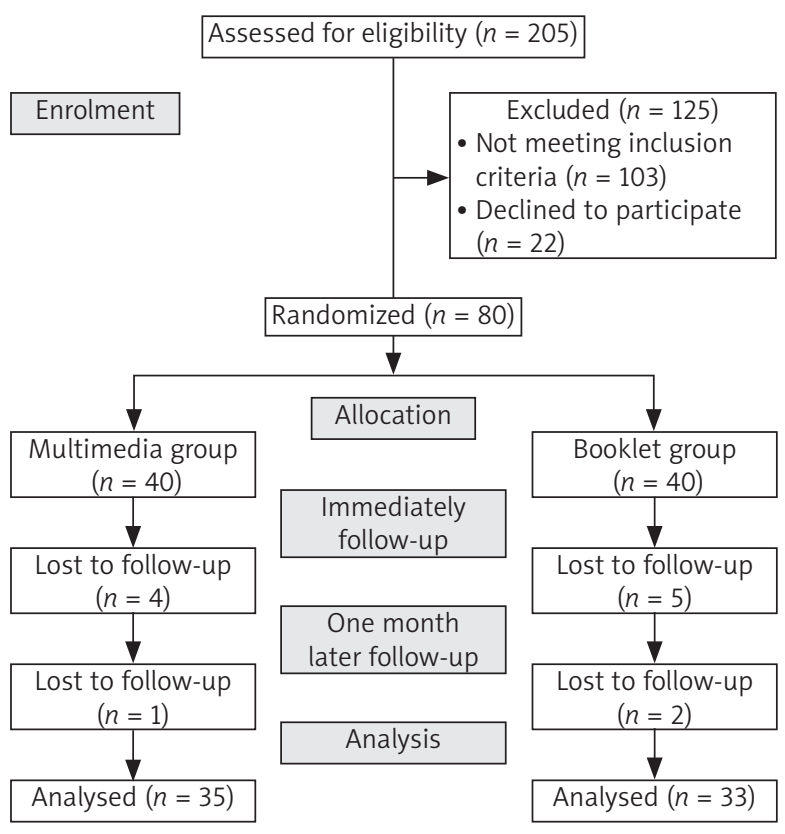

Fig. 1. CONSORT flowchart of study representation

tion. There was no significant difference between the two groups in terms of uncertainty about menopause before the intervention ( $p=0.16$ ) (Table 2). The rANOVA showed no difference between the two groups in terms of uncertainty about menopause $(p=0.34)$. There was a decrease in uncertainty immediately after intervention in both groups, but the score increased one month after intervention, and these changes were statistically significant $(p<0.001)$. There was no interaction between time and groups ( $p=0.20$ ) (Fig. 3). Comparing each time point together, there was no significant difference between the two groups in terms of uncertainty about menopause immediately and one month after intervention $(p<0.05)$ (Table 3, Fig. 3).

\section{Discussion}

In this study, the effect of multimedia and booklet teaching methods on the uncertainty of menopause in postmenopausal women was compared. The results of

Table 1. Baseline sociodemographic characteristics of the two groups

\begin{tabular}{|c|c|c|c|}
\hline Variables & $\begin{array}{l}\text { Multimedia group } \\
\quad(n=35)\end{array}$ & $\begin{array}{l}\text { Booklet group } \\
(n=33)\end{array}$ & $p$-value \\
\hline Age (years), mean (SD) & $49.69(3.40)$ & $50.65(3.20)$ & 0.23 \\
\hline Education (years), mean (SD) & $8.45(3.72)$ & $9.94(4.15)$ & 0.12 \\
\hline \multicolumn{3}{|l|}{ Work status, $n(\%)$} & \multirow{4}{*}{0.79} \\
\hline Housewife & $24(72.7)$ & $23(65.7)$ & \\
\hline Employee & $4(12.1)$ & $6(17.1)$ & \\
\hline Retired & $5(15.2)$ & $6(17.1)$ & \\
\hline Age of menarche (years), mean (SD) & $13.24(1.76)$ & $13.97(1.75)$ & 0.09 \\
\hline Number of children, mean (SD) & $3.18(0.91)$ & $3.05(1.28)$ & 0.64 \\
\hline Last menstrual period (months ago), mean (SD) & $29.16(19.27)$ & $34.62(20.80)$ & 0.39 \\
\hline
\end{tabular}




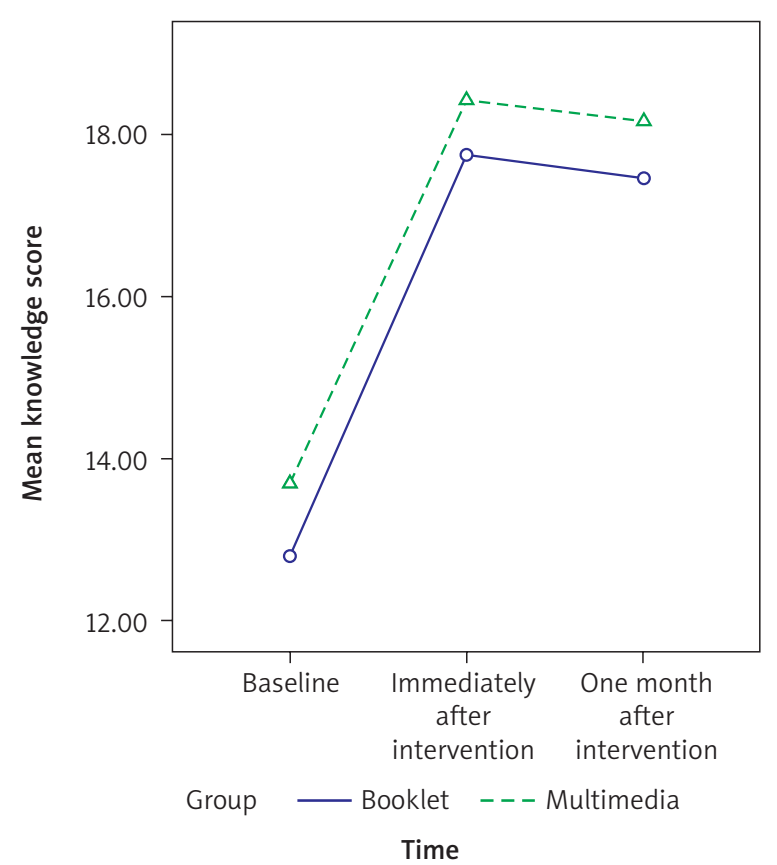

Fig. 2. Comparison of the score of knowledge about menopause over time in booklet and multimedia groups

this study demonstrated a significantly positive effect of both multimedia and booklet educational methods on decreasing menopause uncertainty scores. Although no study has been done to compare the impact of these two educational methods on uncertainty so far, other studies show the impact of other educational methods on the uncertainty variable in menopause. One study by Lemaire et al., which investigated the effect of educational intervention through giving lectures on the reformation of uncertainty in postmenopausal women, revealed that high levels of uncertainty in menopause can be reduced by educational intervention using the lecture method [14].

Other studies have also designed interventions using some other educational methods to reduce uncertainty in various illnesses. For example, Lee et al. performed a study on patients with sudden hearing loss. Their findings demonstrated that oral educational interventions along with written presentation of informa-

Table 2. Comparison of the score of knowledge about menopause over time in the multimedia and booklet groups

\begin{tabular}{lccc}
\hline Time & $\begin{array}{c}\text { Multimedia } \\
\text { group } \\
\text { Mean (SD) }\end{array}$ & $\begin{array}{c}\text { Booklet } \\
\text { group } \\
\text { Mean (SD) }\end{array}$ & p-value* \\
\hline Baseline & $12.78(3.19)$ & $13.68(4.25)$ & 0.32 \\
\hline $\begin{array}{l}\text { Immediately after } \\
\text { intervention }\end{array}$ & $17.75(4.79)$ & $18.42(3.60)$ & 0.51 \\
\hline $\begin{array}{l}\text { One month after } \\
\text { intervention }\end{array}$ & $17.45(3.61)$ & $18.17(3.42)$ & 0.40 \\
\hline \begin{tabular}{l} 
Repeated measurement ANOVA showed between-group $p$-value $=0.34$ \\
\hline
\end{tabular}
\end{tabular}

and within-group p-value $<0.001 ;{ }^{*}$ independent samples $t$-test was used

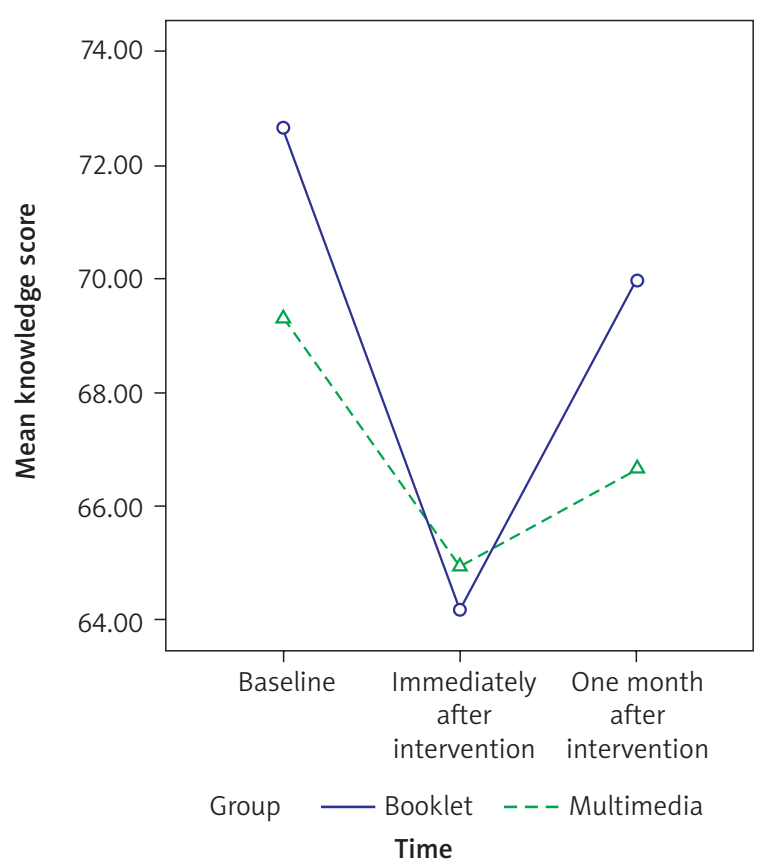

Fig 3. Comparison of the score of uncertainty about menopause over time in booklet and multimedia groups

tion could positively affect the recognition of the illness and reduce uncertainty of it [30].

The results of this study showed that there was no significant difference between the mean score of uncertainty in menopause in the pre-intervention stage and one month after the intervention $(p=0.12)$. This finding means that the uncertainty score, despite increasing immediately after the intervention, was reduced one month after the intervention and reached the same level as before the intervention. This finding could indicate that educational interventions to reduce uncertainty in postmenopausal women should be designed for a longer and continuous period of time.

The results of this study also showed a significantly positive effect of both educational methods on raising awareness among women. The findings of Badiei et al. are consistent with the results of this study. They reported that both booklet and electronic continuous education enhanced the nurses' knowledge of diabetes

Table 3. Comparison of the score of uncertainty about menopause over time in the multimedia and booklet groups

\begin{tabular}{lccc}
\hline Time & $\begin{array}{c}\text { Multimedia } \\
\text { group } \\
\text { Mean (SD) }\end{array}$ & $\begin{array}{c}\text { Booklet } \\
\text { group } \\
\text { Mean (SD) }\end{array}$ & $p$-value* \\
\hline Baseline & $69.31(10.06)$ & $72.66(9.52)$ & 0.16 \\
\hline $\begin{array}{l}\text { Immediately after } \\
\text { intervention }\end{array}$ & $64.94(9.04)$ & $64.18(11.81)$ & 0.76 \\
\hline $\begin{array}{l}\text { One month after } \\
\text { intervention }\end{array}$ & $66.65(10.56)$ & $69.96(8.96)$ & 0.16 \\
\hline
\end{tabular}

Repeated measurement ANOVA showed between-group $p$-value $=0.3$ and within-group p-value $<0.001 ;{ }^{*}$ independent samples $t$-test was used 
updates [31]. Rostom et al. reported that both booklet and computer instructional methods enhanced the knowledge and attitude of postmenopausal women toward decision-making on the acceptance of hormone replacement therapy [32].

According to the results of this study, there was no significant difference between knowledge and uncertainty scores in menopause immediately after intervention and one month after intervention in both groups $(p>0.05)$. This finding suggests that the use of booklet and multimedia methods do not have a particular priority, and both are similar in increasing awareness and reducing uncertainty in menopause.

Although no study has been done yet to compare the effect of these two methods on the uncertainty among women of transition to menopause, the results of studies that have been reviewed in other areas can be considered. One can mention the study by Murray et al., who reported that there was no significant difference in satisfaction, anxiety, utilisation of health care resources, and general health status of women in the post-menopausal period between the booklet and multimedia methods [33]. In their study, Dilles et al., with the aim of comparing the effect of computer-based and booklet educational methods on the knowledge and self-care of cardiac patients, observed that there was no significant difference between the two groups in terms of knowledge and self-care scores [34]. Likewise, in the study by Jamalivand et al., which aimed at comparing the two methods on the knowledge and self-esteem of pregnant women, the results did not show significant differences between the two groups in terms of knowledge and self-esteem [35]. However, other studies have revealed contradictory results in comparing the effects of the two methods. For instance, Abbasi et al. conducted a study on pregnant women, and the findings demonstrated higher effectiveness of the multimedia method than the booklet in improving women's knowledge about labour pain management [36]. The results of the study by Mohamadirizi et al. also showed that an e-learning program increased the level of satisfaction of nulliparous women in the postpartum period more than the booklet method [37].

It seems that the impact of the multimedia and booklet methods can be due to several factors, including the age of the target group or the educational content concerned. One of the reasons for the difference in the results of this study with those of similar studies can be due to the difference in the age of the participants. The average age of the subjects in this study was 50.18 years, while in similar studies the average age was about 25-26 years. This can affect the interest of individuals in using advanced technologies, including computers.

It should also be noted that each medium has its own advantages and disadvantages, which can affect the quality of teaching. The booklet has the advantage of ease of use in a variety of spatial and temporal conditions, while the multimedia method, despite the possibility of using sound and image and its greater attractiveness, due to the inability to use it in all conditions or lack of skill in using it, can have fewer positive outcomes among people [38].

One of the limitations of the current study is that it was conducted on women in Gonabad, which limits the generalisability of the results. Therefore, it is suggested that future studies be conducted on other populations. Also, since the findings of this study did not show a significant difference between the effect of the multimedia and booklet educational methods on uncertainty in menopause, it is suggested that other studies find more effective educational methods in this respect.

\section{Conclusions}

According to the findings of this study, the high level of perceived uncertainty associated with menopause is reduced by both multimedia and booklet methods. Because the findings of this study did not show a significant difference between the two educational methods on decreasing uncertainty in menopause, instead of using the multimedia method, which is costly and complex and requires IT professionals to design it, the booklet method can be used.

\section{Acknowledgements}

This study was sponsored by Gonabad University of Medical Sciences. The authors are grateful for this support. Special thanks also go to the middle-aged women for their participation.

\section{Disclosure}

The authors report no conflict of interest.

\section{References}

1. Morowatisharifabad MA, Yoshany N, Bahri N, et al. Effects of an educational intervention based on the multi-theory model on promoting the quality of life in postmenopausal women: a protocol. Menopause Rev 2019; 18: 153-160.

2. Moral E, Delgado JL, Carmona F, et al. The impact of genitourinary syndrome of menopause on well-being, functioning, and quality of life in postmenopausal women. Menopause 2018; 25: 1418-1423.

3. Kalra B, Agarwal S, Magon S. Holistic care of menopause: Understanding the framework. J Midlife Health 2012; 3: 66-69.

4. Monteleone P, Mascagni G, Giannini A, et al. Symptoms of menopause - global prevalence, physiology and implications. Nat Rev Endocrinol 2018; 14: 199-215.

5. Eraslan D, Ertekin E, Ertekin BA, Oztürk O. Treatment of insomnia with hypnotics resulting in improved sexual functioning in post-menopausal women. Psychiatr Danub 2014; 26: 353-357. 
6. Bahri N, Sajjadi M, Sadeghmoghadam L. The Relationship between Management Strategies for Menopausal Symptoms and Women's Decision-Making Styles during Menopausal Transition. Menopause Rev 2019; 18: 174-179.

7. Mishel MH, Germino BB, Gil KM, et al. Benefits from an uncertainty management intervention for African-American and Caucasian older long-term breast cancer survivors. Psychooncology 2005; 14: 962-978.

8. Sajjadi M, Rassouli M, Bahri N, Mohammadipoor F. The correlation between perceived social support and illness uncertainty in people with human immunodeficiency virus/acquired immune deficiency syndrome in Iran. Indian J Palliat Care 2015; 21: 231-235.

9. Bahri N, Latifnejad Roudsari R, Azimi Hashemi M. "Adopting self-sacrifice": how Iranian women cope with the sexual problems during the menopausal transition? An exploratory qualitative study. J Psychosom Obstet Gynaecol 2017; 38: 180-188.

10. Bahri N, Latifnejad Roudsari R. "Moving from uncertainty toward acceptance": a grounded theory study on exploring Iranian women's experiences of encountering menopause. J Psychosom Obstet Gynecol 2020, in press. DOI: 10.1080/0167482X.2019.1678018

11. Walter CA. The psychosocial meaning of menopause: women's experiences. J Women Aging 2000; 12: 117-131.

12. Banister EM. Women's midlife confusion: "Why am I feeling this way?". Issues Ment Health Nurs 2000; 21: 745-764.

13. Hoga L, Rodolpho J, Gonçalves B, Quirino B. Women's experience of menopause: a systematic review of qualitative evidence. JBI Database System Rev Implement Rep 2015; 13: 250-337.

14. Lemaire GS, Lenz ER. Perceived uncertainty about menopause in women attending an educational program. Int J Nurs Stud 1995; 32: 39-48.

15. Hvas L, Reventlow S, Jensen HL, Malterud K. Awareness of risk of osteoporosis may cause uncertainty and worry in menopausal women. Scand J Public Health 2005; 33: 203-207.

16. Moreira AC, Silva AM, Santos MS, Sardão VA. Phytoestrogens as alternative hormone replacement therapy in menopause: What is real, what is unknown. J Steroid Biochem Mol Biol 2014; 143: 61-71.

17. Hansen KA, Eyster KM. What happened to WHI: menopausal hormonal therapy in 2012. Clin Obstet Gynecol 2012; 55: 706-712.

18. Pai HC, Lee S, Tseng YH. A correlative study of the uncertainty in the climacteric women. Hu Li Za Zhi 2004; 51: 45-52.

19. Morgan P, Merrell J, Rentschler D, Chadderton H. Uncertainty during perimenopause: perceptions of older first-time mothers. J Adv Nurs 2012; 68: 2299-2308

20. Sharif SP, Ahadzadeh AS, Perdamen HK. Uncertainty and quality of life of Malaysian women with breast cancer: Mediating role of coping styles and mood states. Appl Nurs Res 2017; 38: 88-94

21. Lasker JN, Sogolow ED, Olenik JM, et al. Uncertainty and liver transplantation: women with primary biliary cirrhosis before and after transplant. Women Health 2010; 50: 359-375.

22. Hoth KF, Wamboldt FS, Strand M, et al. Prospective impact of illness uncertainty on outcomes in chronic lung disease. Health Psychol 2013; 32: 1170-1174.

23. Lemaire GS. More than just menstrual cramps: symptoms and uncertainty among women with endometriosis. J Obstet Gynecol Neonatal Nurs 2004; 33: 71-79.

24. Bahri N, Bagheri S, Erfani M, et al. The Comparison of Workshop-Training and Booklet-Offering on Knowledge, Health Beliefs and Behavior of Breastfeeding after Delivery. IJOGI 2013; 15: 14-22.

25. O'Halloran P, Scott D, Reid J, Porter S. Multimedia psychoeducational interventions to support patient self-care in degenerative conditions: A realist review. Palliat Support Care 2015; 13: 1473-1486.

26. Huang JP, Chen HH, Yeh ML. A comparison of diabetes learning with and without interactive multimedia to improve knowledge, control, and selfcare among people with diabetes in Taiwan. Public Health Nurs 2009; 26: 317-328.

27. Mohamadirizi S, Bahadoran P, Fahami F. Comparison between the Impacts of E-Learning and Booklet Education on Nulliparous Women's Satisfaction about Postpartum Care. IJOGI. Summer 2013; 16: 1-8.

28. CONSORT 2010 checklist of information to include when reporting a randomised trial. Available at: http://consort-statement.org/media/ defult/downloads/COSORT 2010 Checklist.pdf (access: 30 Jun 2018).

29. http://webda.mums.ac.ir/index.php?option=com_content\&view=article\&id =6078 (access: 30 March 2018).
30. Lee $\mathrm{YH}$, Wang $\mathrm{HL}$, Li CY, et al. Exploring the effectiveness of a health education program on the stimuli frame and on uncertainty in patients with sudden hearing loss. Hu Li Za Zhi 2014; 61: 36.

31. Badiei M, Gharib M, Zolfaghari M, Mojtahedzadeh R. Comparing nurses' knowledge retention following electronic continuous education and educational booklet: a controlled trial study. Med J Islam Repub Iran 2016; $30: 364$

32. Rostom A, O'Connor A, Tugwell P, Wells G. A randomized trial of a computerized versus an audio-booklet decision aid for women considering post-menopausal hormone replacement therapy. Patient Educ Couns 2002; 46: 67-74.

33. Murray E, Davis H, Tai SS, et al. Randomised controlled trial of an interactive multimedia decision aid on hormone replacement therapy in primary care. BMJ 2001; 323: 490-493.

34. Dilles A, Heymans V, Martin S, et al. Comparison of a computer assisted learning program to standard education tools in hospitalized heart failure patients. Eur J Cardiovasc Nurs 2011; 10: 187-193.

35. Jamalivand S, Mohammad-Alizadeh Charandabi S, Mirghafourvand M. Comparing the Effect of Electronic Software and Training Booklet on Maternal Self-Confidence and Awareness About Newborn Care: A Randomized Controlled Clinical Trial, Iran Red Crescent Med J 2017; 19: e44152.

36. Abbasi P, Mohammad-Alizadeh Charandabi S, Mirghafourvand M. Comparing the effect of e-learning and educational booklet on the childbirth self-efficacy: a randomized controlled clinical trial. J Matern Fetal Neonatal Med 2018; 31: 644-650.

37. Mohamadirizi S, Bahadoran P, Fahami F. Comparison between the impacts of e-learning and booklet education on nulliparous women's satisfaction about postpartum care. IJOG 2013; 16: 1-8.

38. Zarshenas L, Keshavarz T, Momennasab M, Zarifsanaiey N. Interactive Multimedia Training in Osteoporosis Prevention of Female High Schoo Students: An Interventional Study. Acta Med Iran 2017; 55: 514 